Final Draft of Joseph, S., Lid, V., \& Suthers, D. (2007). Transcendent Communities. In C. Chinn, G. Erkens \& S. Puntambekar (Eds.), The Computer Supported Collaborative Learning (CSCL) Conference 2007 (pp. 317-319). New Brunswick: International Society of the Learning Sciences.

\title{
Transcendent Communities
}

\author{
Sam Joseph, Viil Lid, Dan Suthers, \\ University of Hawaii, 1380 East-West Road Honolulu, HI \\ Email: srjoseph@hawaii.edu,viil@hawaii.edu,suthers@hawaii.edu
}

\begin{abstract}
Online communities are potential arenas for informal and lifelong learning. Even though technology fosters internal sharing and collaboration in online communities, it also presents excessively strong external boundaries. These silo-like structures lead to fragmentation, counteracting cross-community collaboration and interdisciplinary learning. We are revising our own online community software to support a particular sociotechnical pattern: the emergence of "transcendent communities"- networks of participation that transcend collections of related but distinct communities. In order to understand such inter-community activity we have developed a theoretical analysis of the basis for individual action and how this action can lead to value for the larger community. Investigating the relationships between individual action, social affordances of the technology and group identities will help us to design for functionality and for meaning.
\end{abstract}

\section{Introduction}

Like traditional communities, online communities have their own identities, norms, and goals, and several of a community's objectives may be shared with one or more related communities. Together these communities and their purpose may benefit from sharing information, coordinating events, and collaborating towards their common goals while still maintaining their distinctions as individual communities. For example, groups in an online teacher community we support (hnlc.org) have to share with each other, yet each group needs its own virtual "place" to conduct its work. It has been challenging to provide these nested and overlapping groups with the identity and space they need while maintaining awareness and sharing of resources at all levels. A similar problem exists in postsecondary education. Students experience courses as silos, isolated from each other. Unfortunately the silo approach can inhibit the sorts of collaboration that can be most conducive to learning (Derry \& Fischer, 2005). For example, students and faculty in our own interdisciplinary Communication and Information Science program (www.hawaii.edu/cis/) participate in multiple nested and overlapping groups and organizational units and are members of a larger community. ${ }^{1}$ Yet, this fact is not well supported by current online learning environments, including our own (disCourse.ics.hawaii.edu).

Although fragmentation is part of the problem, complete unification under one identity, goal or technological space is not the solution. Even though the constituent collectives share some goals, they are likely to have additional objectives that are not shared and that make them unique. These goals are a part of each collective's identity that attracts and ties the members to the collective and that in turn makes up parts of their own identities (Tajfel \& Turner, 1986). Fragmentation into smaller community sites is also a problem for large community sites (Bruckman \& Jensen, 2002). The desire to maintain existing identities can prevent smaller communities from merging into larger ones. Successful coordination between communities forms only by the deliberate and dedicated effort of those who span their boundaries (Levina \& Vaast, 2005).

When two or more collectives share concerns, there is potential for an alternate solution: the formation of a community that transcends formal boundaries and has greater potential to reach the critical mass (Markus, 1987) needed to achieve a new level of collective activity. This approach resolves the fundamental tension that groups need to be small and have a sense of shared intimacy, yet also need to become connected to a larger set of likeminded groups in order to progress. To achieve this, flexible technological boundaries are needed similar to those in traditional communities (Barth, 1981; Cohen, 1985) where boundary-spanning activities may take place. A balance is needed between global connectivity and distinct groups, requiring support for permeable boundaries of participation that lead to communities transcending associated collectives without violating the integrity of each collective unit. We need to overcome the tendency of technology to present stark digital boundaries that discourage communication (Postmes, Spears, \& Lea, 1998).

\footnotetext{
${ }^{1}$ This paper uses the terms collective generically for social entities, group for networks of individuals who regularly interact (e.g., workgroups), community for people sharing symbolically constructed identities, and organization for collectives that are hierarchically structured to fill a functional need in society.
} 
Final Draft of Joseph, S., Lid, V., \& Suthers, D. (2007). Transcendent Communities. In C. Chinn, G. Erkens \& S. Puntambekar (Eds.), The Computer Supported Collaborative Learning (CSCL) Conference 2007 (pp. 317-319). New Brunswick: International Society of the Learning Sciences.

\section{From Activity Cycles to Communities}

Since transcendent communities will arise only based on the actions of individuals, it is essential to understand the basis for individual action and how it can lead to value for the community. One way of looking at individual activity is through the lens of the participation-reification duality (Wenger, 1998). In this view, acts of participation are intrinsically bound up with reifications that both frame and leave a trace of that act. When participants come across traces of previous participation, further participation may occur leading to further reification. Ideally a virtuous cycle occurs as participation levels increase along with a well-organized set of valuable resources. Figure 1 displays an activity cycle of find $\rightarrow$ care $\rightarrow$ act $\rightarrow$ persist with the various factors that affect each stage of the cycle, bridging the realms of

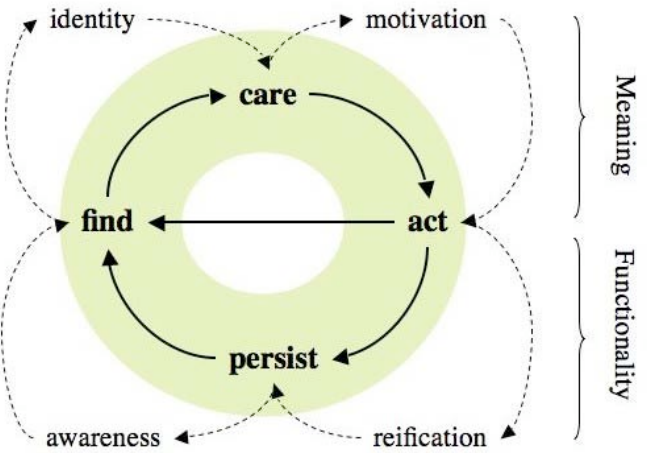

Figure 1. Activity cycle and related factors functionality and meaning. We posit this cycle of online activity as a basis for analyzing how the confluence of participation and identity in a technological environment increases value for the community. Any participation involves a physical reification, but the persistence of that reification is a function of the medium in which it is expressed. Media may be designed to increase the likelihood of capture and persistence of individual activity as a potential source of value for others. A persistent trace of participation can become the basis for subsequent participation in a two-step process. First of all, a given reification has to be found, by accident or intent. Again, media may be designed such that past reifications are available for chance or intentional discovery, by promoting awareness and associative access. Second, given that a reification has been found by an individual, there arises the question of whether this individual will care enough to act on it. Caring critically involves identity and acting involves motivation, which are functions of the history of previous interactions. For example, if an individual encounters a reification of prior participation by a person she perceives as belonging to some shared community or having common goals, then the individual may act on this reification, continuing the cycle of activity. In summary, in order to foster interactions between individuals that will lead to collective value, this analysis guides design to ensure as best as possible that relevant reifications of activity are persisted, that these traces can be easily found, and that individuals can perceive group and individual identities associated with the reifications and their potential value so that potential participants will care enough to further participate.

In the ideal environment the medium and its social-technical affordances will maximize the likelihood of each of the individual processes that allow this cycle to be continued and connected across many individuals. Figure 2 shows how the persistence of reifications connects the activities of different individuals. Multiple interconnected activity cycles can lead to a group of repeatedly participating individuals who derive benefit from access to the aggregated resources, as well as occasional participants who perhaps contribute less, but benefit all the same. Given an open environment the number of participants is unlimited, as is the value that may be created. However in reality there will be pools of more tightly interconnected reifications, as there will be sets of individuals who more frequently interact with each other's reifications, creating and sustaining groups and communities.

Individuals may participate in multiple groups and perceive themselves as belonging to different degrees to multiple communities. We can see a group as a network of interacting individuals and a community as a group that is distinguished through common identity. In order to promote the creation of emergent communities we need to encourage more than just a few trajectories that go across the tight networks - we need to encourage "boundary spanning" as a natural activity.

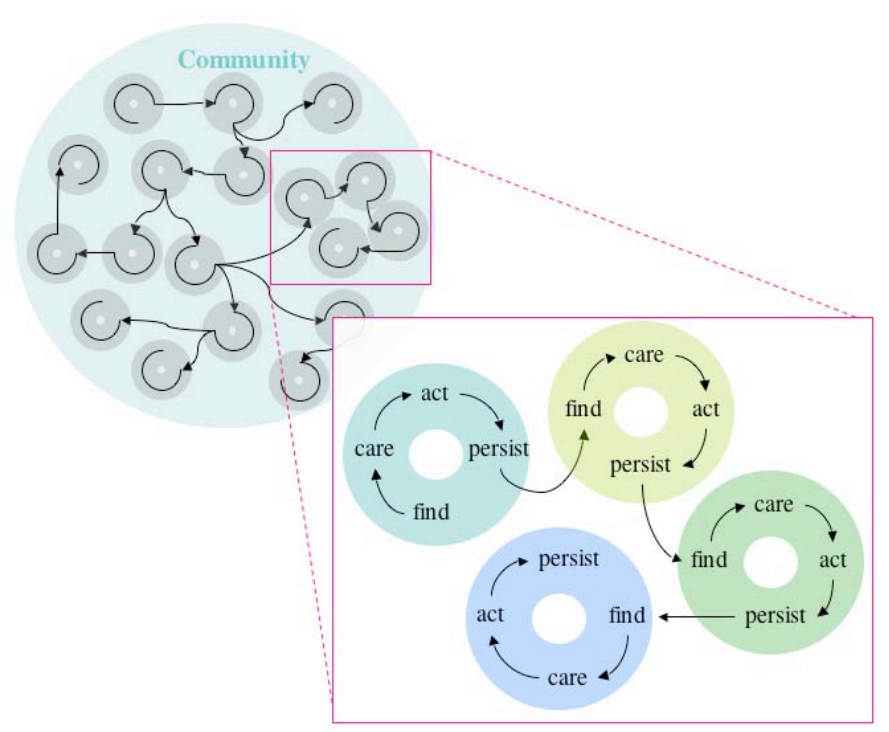

Figure 2. Persistence connecting activity cycles 
Final Draft of Joseph, S., Lid, V., \& Suthers, D. (2007). Transcendent Communities. In C. Chinn, G. Erkens \& S. Puntambekar (Eds.), The Computer Supported Collaborative Learning (CSCL) Conference 2007 (pp. 317-319). New Brunswick: International Society of the Learning Sciences.



Figure 3. Pathways connecting communities

Boundary spanning can be accomplished both by the movement of individuals and the sharing of objects. Figure 3 displays the creation of a sub-domain or boundary domain between two communities as participants are enticed to participate in activity cycles involving each others' reifications.

\section{Conclusion}

offering new technologies are collaboration, but increased connectivity must be balanced with the need for maintaining intimate communities. If transdisciplinary education is to succeed, we need to enable trans-boundary participation without posing a threat to the involved communities' identities. In order to address this we need to understand the basis for individual action, how this action can lead to value for the larger community, and how social affordances of technology can amplify both of these. The activity cycles reveal how individual action is influenced by factors of both functionality and meaning that should be addressed by design: awareness and access, portrayal of identity, goal-relevance, and conversion of activity into value for others. We can then use this understanding of individual action to bridge to the community level, investigating how sociotechnical affordances enhance or inhibit the productive entanglements between individual trajectories of activity within and across communities. Analysis of the activity cycles should help us better understand the operations of boundary spanners and boundary objects; ultimately allowing us to design sociotechnical affordances that facilitate the achievement of critical mass through multiple inter-community interactions. Our ongoing work seeks to contribute towards a general understanding of design for transcendent communities: larger spheres of common identity and social capital that arise from interactions between individuals who participate in collections of related communities.

\section{References}

Barth, F. (1981). Process and Form in Social Life. London: Routledge \& Kegan Paul.

Bruckman, A. S., \& Jensen, M. S. (2002). The mystery of the death of MediaMOO: Seven years of evolution of an online community in building virtual communities. In A. K. Renninger \& W. Shumar (Eds.), Building Virtual Communities (pp. 21-33). Cambridge: Cambridge University Press.

Cohen, A. P. (1985). The symbolic construction of community: Tavistock Publications.

Derry, S. J., \& Fischer, G. (2005). Transdisciplinary Graduate Education, American Educational Research Association. Montreal, Canada.

Levina, N., \& Vaast, E. (2005). The Emergence of Boundary Spanning Competence in Practice: Implications for Implementation and Use of Information Systems. MIS Quarterly, 29(2), 335-363.

Markus, M. L. (1987). Toward a critical mass theory of interactive media: Universal access, interdependence and diffusion. Communication Research, 14, 491-511.

Postmes, T., Spears, R., \& Lea, M. (1998). Breaching or building social boundaries? SIDE-effects of computermediated communication. Communication Research, 25(6), 689-715.

Tajfel, H., \& Turner, J. C. (1986). The social identity theory of inter-group behavior. In S. Worchel \& L. W. Austin (Eds.), Psychology of Intergroup Relations. Chicago: Nelson-Hall.

Wenger, E. (1998). Communities of Practice: Learning, Meaning and Identity. Cambridge: Cambridge University Press.

\section{Acknowledgments}

This work was supported by the National Science Foundation under RSI award 0100393. Kar-Hai Chu assisted with exploratory analyses. 